\title{
Automorphisms of a certain subalgebra of the upper triangular matrix algebra
}

\author{
Özkay Özkan*(D), Mustafa Akkurt (D) \\ Gebze Technical University Department of Mathematics 2254. Sk. Gebze 41400, Kocaeli, Turkey
}

\begin{abstract}
For a commutative ring $R$ with unity, the $R$-algebra of strictly upper triangular $n \times n$ matrices over $R$ is denoted by $N_{n}(R)$, where $n$ is a positive integer greater than 1 . For the identity matrix $I, \alpha \in R, A \in N_{n}(R)$, the set of all elements $\alpha I+A$ is defined as the scalar upper triangular matrix algebra $S T_{n}(R)$ which is a subalgebra of the upper triangular matrices $T_{n}(R)$. In this paper, we investigate the $R$-algebra automorphisms of $S T_{n}(R)$. We extend the automorphisms of $N_{n}(R)$ to $S T_{n}(R)$ and classify all the automorphisms of $S T_{n}(R)$. We generalize the results of Cao and Wang and prove that not all automorphisms of $S T_{n}(R)$ can be extended to the automorphisms of $T_{n}(R)$.
\end{abstract}

Mathematics Subject Classification (2010). 15A30, 16S50, 08A35

Keywords. triangular matrix algebra, upper triangular matrix algebra, automorphisms

\section{Introduction}

Kezlan [3] proved that: "If $R$ is a commutative ring with unity, then every $R$-algebra automorphism of the algebra of upper triangular $n \times n$ matrices over $R$ is inner ". Coelho [2] extended Kezlan's result for any ring $R$. Cao and Wang [1] classified all the automorphisms of strictly upper triangular matrix algebras over a commutative ring $R$. In this work, we would like to generalize these results and determine how such central automorphisms exist on $S T_{n}(R)$ but not on $T_{n}(R)$.

Let $R$ be a commutative ring with unity and $N_{n}(R)$ be the algebra of all $n \times n$ strictly upper triangular matrices over $R$, where $n$ is a positive integer greater than 1 .

Define $S T_{n}(R)$ with unity matrix $I$,

$$
S T_{n}(R)=\left\{\alpha I+A: \alpha \in R, A \in N_{n}(R)\right\} .
$$

If $X \in S T_{n}(R)$, we may write $X=\alpha I+A=\alpha I+\sum_{i<j} a_{i j} E_{i j}$ where $\alpha \in R$ and $A \in N_{n}(R) . S T_{n}(R)$ is a subalgebra of $T_{n}(R)$ and let us call the scalar upper triangular matrix algebra. The standard matrix units are the matrices $E_{i j}$ with a 1 at $(i, j)$ position and zero elsewhere. Clearly, the set of matrices $\left\{E_{i j}: 1 \leq i<j \leq n\right\}$ form a basis of

\footnotetext{
*Corresponding Author.

Email addresses: ozkayoz@yahoo.com (Ö. Özkan), makkurt@gtu.edu.tr (M. Akkurt)

Received: 17.01.2019; Accepted: 27.08.2019
} 
$N_{n}(R)$. For $n \geq 4$, consider $c=\left(c_{2}, \ldots, c_{n-2}\right) \in R^{n-3}$. Y.Cao and J. Wang [1] defined the central automorphisms of $N_{n}(R), \mu_{c}: N_{n}(R) \rightarrow N_{n}(R)$ such that for any $A \in N_{n}(R)$

$$
\mu_{c}(A)=A+\left(\sum_{k=2}^{n-2} a_{k, k+1} c_{k}\right) E_{1 n} .
$$

With respect to the basis of $N_{n}(R)$, automorphisms can be written as:

$$
\mu_{c}\left(E_{i, i+1}\right)=E_{i, i+1}+c_{i} E_{1 n}
$$

for $2 \leq i \leq n-2$ and

$$
\mu_{c}\left(E_{i j}\right)=E_{i j}
$$

for $j \neq i+1$. For any matrix $X \in S T_{n}(R)$, let $S_{1}, S_{2}, \ldots, S_{n-1}$ define the diagonals of $X=\alpha I+\sum_{i<j} a_{i j} E_{i j}$ as follows:

$$
\begin{aligned}
S_{1}= & \left\{a_{12}, a_{23}, \ldots, a_{n-1, n}\right\}, \\
S_{2}= & \left\{a_{13}, a_{24}, \ldots, a_{n-2, n}\right\}, \\
& \vdots \\
S_{n-1}= & \left\{a_{1 n}\right\} .
\end{aligned}
$$

\section{Automorphisms of $S T_{n}(R)$}

There are no invertible elements in $N_{n}(R)$, hence one cannot define an inner automorphism by the usual terminology. For a fixed matrix $X \in T_{n}(R)$ with 1 on the main diagonal, the map $\Theta(Y)=X Y X^{-1}$ is an inner automorphism [1]. The restriction of $\Theta$ on $N_{n}(R)$ is also an automorphism of $N_{n}(R)$.

Moreover, we define the inner automorphism $\theta$ on $S T_{n}(R)$, with a given invertible matrix $B \in T_{n}(R)$ and for any $A \in S T_{n}(R)$ as

$$
\theta(A)=B A B^{-1} \text {. }
$$

In this section, we are going to classify the automorphisms of $S T_{n}(R)$. We first investigate the central automorphisms of $S T_{n}(R)$ in the classification. It is not difficult to see that, for $n \geq 4$,

$$
\mu_{c}: S T_{n}(R) \rightarrow S T_{n}(R)
$$

is an automorphism of $S T_{n}(R)$. To avoid the details of a tedious proof, we use Kezlan's [3] notation. Let

$$
\theta: T_{n}(R) \longrightarrow T_{n}(R)
$$

be an $R$-algebra automorphism of $T_{n}(R)$. Define

$$
\begin{aligned}
\theta\left(E_{k k}\right) & =\left[e_{i j}^{(k)}\right] \text { for } k=1,2, \ldots, n \\
\theta\left(E_{k, k+1}\right) & =\left[a_{i j}^{(k)}\right] \text { for } k=1,2, \ldots, n-1 .
\end{aligned}
$$

The results in [3] show that $a_{k, k+1}^{(k)}$ is a unit with $a_{k, k}^{(k)}=0, e_{k, k}^{(k)}=1$ and $e_{k, k}^{(q)}=0$ for $q \neq k$.

Remark 2.1. In terms of the image of matrix units under $\theta$, it is shown that, $k^{\text {th }}$ diagonal entry of $\theta\left(E_{k k}\right)$ is 1 , while the other diagonal entries are 0 . That is $\theta\left(E_{k k}\right)$ is in the form $\theta\left(E_{k k}\right)=E_{k k}+J_{k}$ where $J_{k}$ is strictly upper triangular. For $k=1, \cdots, n$ we use the fact 
that $\theta\left(E_{k k}\right)$ is the idempotent, that is $\theta\left(E_{k k}\right)=\theta\left(E_{k k}\right) \theta\left(E_{k k}\right)$. Thus as a consequence of $[3], \theta\left(E_{k k}\right)$ has the following form:

$$
\theta\left(E_{k k}\right)=\left[\begin{array}{ccccccc}
0 & \cdots & 0 & e_{1, k}^{(k)} & * & \cdot & * \\
\vdots & \cdots & \vdots & \vdots & \cdot & \cdot & \cdot \\
0 & \cdots & 0 & e_{k-1, k}^{(k)} & * & \cdot & * \\
0 & \cdots & 0 & 1 & e_{k, k+1}^{(k)} & \cdot & e_{k, n}^{(k)} \\
0 & \cdots & 0 & 0 & 0 & \cdot & 0 \\
\vdots & \vdots & \vdots & \vdots & \vdots & \vdots & \vdots \\
0 & . & 0 & 0 & 0 & . & 0
\end{array}\right] .
$$

The following proposition gives us the property of $\theta\left(E_{k k}\right)=\left[e_{i j}^{(k)}\right]$.

Proposition 2.2. Assume that $\theta\left(E_{k k}\right)=\left[e_{i j}^{(k)}\right]$ for $k=1, \ldots, n$. If $i<j$ then for $i=$ $1, \ldots, n-1$

$$
\sum_{k=i}^{j} e_{i j}^{(k)}=0
$$

Proof. It was shown in [3] that

$$
e_{k k}^{(p)}=\left\{\begin{array}{l}
1 \text { if } p=k \\
0 \text { if } p \neq k
\end{array}\right.
$$

Consider $\left(e_{i j}^{(1)}, e_{i j}^{(2)}, \ldots, e_{i j}^{(r)}\right)$ as an $r$-tuple and define $r$-tuple sum as $e_{i j}^{(1)}+e_{i j}^{(2)}+\ldots+e_{i j}^{(r)}$. To use induction on $r$, we start with the case $r=2$. Using the multiplication of matrix units $E_{p p}$ and $E_{p+1, p+1}$ we get

$$
E_{p p} E_{p+1, p+1}=0 .
$$

Since $\theta(0)=0$, in our notation this result can be viewed as:

$$
\left[e_{i j}^{(p)}\right]\left[e_{i j}^{(p+1)}\right]=0 .
$$

For $p=1, \ldots, n-1$,

$$
\left(e_{p, p+1}^{(p)}+e_{p, p+1}^{(p+1)}\right) E_{p, p+1}=0 .
$$

Then we obtain $e_{p, p+1}^{(p)}+e_{p, p+1}^{(p+1)}=0$ for all 2-tuples. Assume our claim is true for the sum of $(r-1)$-tuples. That is

$$
\sum_{i=p}^{p+r-2} e_{p, p+r-2}^{(i)}=0 \text { for } p=1, \ldots,(n-r)+2 .
$$

Use induction on $p$. For $p=1$,

$$
e_{1 r}^{(1)}+e_{1 r}^{(2)}+\ldots+e_{1 r}^{(r-1)}+e_{1 r}^{(r)}=0 .
$$

Now consider each case for $t=1, \ldots,(r-1)$. We know that $E_{t t} E_{r r}=0$. Apply the automorphism $\theta$ to $E_{t t} E_{r r}$, we get

$$
\theta\left(E_{t t} E_{r r}\right)=0 .
$$

For $t=1, \ldots,(r-1)$ the following terms can be obtained. If $t=1$,

$$
\left(e_{1 r}^{(1)}+e_{1 r}^{(r)}+\sum_{i=2}^{r-1} e_{1 i}^{(1)} e_{i r}^{(r)}\right) E_{1 r}=0
$$


For $1<t \leq r-1$,

$$
\left(e_{1 r}^{(t)}+\sum_{i=2}^{r-1} e_{1 i}^{(t)} e_{i r}^{(r)}\right) E_{1 r}=0
$$

By using the sum

$$
\theta\left(E_{11} E_{r r}+E_{22} E_{r r}+\ldots+E_{(r-1),(r-1)} E_{r r}\right)=0
$$

we get

$$
e_{1 r}^{(1)}+e_{1 r}^{(2)}+\ldots+e_{1 r}^{(r-1)}+e_{1 r}^{(r)}+\sum_{j=2}^{r-1}\left(\sum_{i=1}^{r-1} e_{1 j}^{(i)} e_{j r}^{(r)}\right)=0 .
$$

By the assumption we have, for $j=2, \ldots, r-1$, that

$$
\sum_{j=2}^{r-1}\left(\sum_{i=1}^{r-1} e_{1 j}^{(i)} e_{j r}^{(r)}\right)=0
$$

Also the calculations on $\theta\left(E_{i i}\right)$, in (2.1), can be used to deduce the result,

$$
e_{1 r}^{(1)}+e_{1 r}^{(2)}+\ldots+e_{1 r}^{(r-1)}+e_{1 r}^{(r)}=0 .
$$

Assume that our assumption is true for $p=q$, that is

$$
e_{q, q+r-1}^{(q)}+e_{q, q+r-1}^{(q+1)}+\ldots+e_{q, q+r-1}^{(q+r-2)}+e_{q, q+r-1}^{(q+r-1)}=0 .
$$

Now consider the case $p=q+1$. We want to prove that

$$
e_{q+1, q+r}^{(q+1)}+e_{q+1, q+r}^{(q+2)}+\ldots+e_{q+1, q+r}^{(q+r-1)}+e_{q+1, q+r}^{(q+r)}=0 .
$$

Apply the automorphism $\theta$ to $E_{t t} E_{q+1, q+1}$ for $t=1, \ldots, q$ we get

$$
\theta\left(E_{t t} E_{q+1, q+1}\right)=0 \text {. }
$$

For $t=q+1$,

$$
\left(e_{q+1, q+r}^{(q+1)}+e_{q+1, q+r}^{(q+r)}+\sum_{i=q+2}^{q+r-1} e_{q+1, i}^{(q+1)} e_{i, q+r}^{(q+r)}\right) E_{q+1, q+r}=0 .
$$

For $q+1<t<q+r$,

$$
\left(e_{q+1, q+r}^{(t)}+\sum_{i=t}^{q+r-1} e_{q+1, i}^{(t)} e_{i, q+r}^{(q+r)}\right) E_{q+1, q+r}=0 .
$$

Consider the following sum:

$$
\theta\left(E_{q+1, q+1} E_{q+r, q+r}+E_{q+2, q+2} E_{q+r, q+r}+\ldots+E_{q+r-1, q+r-1} E_{q+r, q+r}\right)=0,
$$

then we have

$$
e_{q+1, q+r}^{(q+1)}+e_{q+1, q+r}^{(q+2)}+\ldots+e_{q+1, q+r}^{(q+r-1)}+e_{q+1, q+r}^{(q+r)}+\sum_{j=q+2}^{q+r-1}\left(\sum_{i=q+1}^{q+r-1} e_{q+1, j}^{(i)} e_{j, q+r}^{(q+r)}\right)=0 .
$$

By the assumption we have

$$
\sum_{j=q+2}^{q+r-1}\left(\sum_{i=q+1}^{q+r-1} e_{q+1, j}^{(i)} e_{j, q+r}^{(q+r)}\right)=0
$$

and this implies

$$
e_{q, q+r-1}^{(q)}+e_{q, q+r-1}^{(q+1)}+\ldots+e_{q, q+r-1}^{(q+r-2)}+e_{q, q+r-1}^{(q+r-1)}=0 .
$$

Thus the proof is completed. 
Lemma 2.3. Let $R$ be a commutative ring with identity and $\theta$ be an $R$-algebra automorphism of $S T_{n}(R)$. If $n=2,3$ then $\theta$ is an inner automorphism $\eta_{D}$ for some $D \in T_{n}(R)$.

Proof. For the automorphism $\theta: S T_{n}(R) \rightarrow S T_{n}(R)$ as given in Remark 2.1, let

$$
\begin{aligned}
\theta\left(E_{k k}\right) & =\left[e_{i j}^{(k)}\right] \text { for } k=1,2, \ldots, n \\
\theta\left(E_{k, k+1}\right) & =\left[a_{i j}^{(k)}\right] \text { for } k=1,2, \ldots, n-1 .
\end{aligned}
$$

Recall that all the diagonal entries $a_{i i}^{(k)}$ of $\theta\left(E_{k, k+1}\right)$ are zero and

$$
e_{q q}^{(p)}=\left\{\begin{array}{cc}
1 & \text { if } p=q \\
0 & \text { otherwise. }
\end{array}\right.
$$

Also $a_{12}^{(1)}, a_{23}^{(2)}, \ldots, a_{n-1, n}^{(n-1)}$ are all units.

For $n=2$, consider $A \in S T_{n}(R)$ where

$$
A=\left[\begin{array}{ll}
\alpha & b \\
0 & \alpha
\end{array}\right]
$$

for $\alpha, b \in R$. Every matrix $A \in S T_{2}(R)$ can be written as linear combination of matrix units. That is,

$$
A=\alpha E_{11}+b E_{12}+\alpha E_{22} .
$$

Applying $\theta$ to $A$, we get

$$
\theta(A)=\alpha \theta\left(E_{11}\right)+b \theta\left(E_{12}\right)+\alpha \theta\left(E_{22}\right) .
$$

By using the notation of Remark 2.1,

$$
\begin{aligned}
& \theta\left(\alpha E_{11}\right)=\left(\begin{array}{cc}
\alpha & \alpha e_{12}^{(1)} \\
0 & 0
\end{array}\right) \\
& \theta\left(b E_{12}\right)=\left(\begin{array}{cc}
0 & b a_{12}^{(1)} \\
0 & 0
\end{array}\right) \\
& \theta\left(\alpha E_{22}\right)=\left(\begin{array}{cc}
0 & \alpha e_{12}^{(1)} \\
0 & \alpha
\end{array}\right) .
\end{aligned}
$$

As a result,

$$
\theta(A)=\left[\begin{array}{cc}
\alpha & \alpha e_{12}^{(1)}+b a_{12}^{(1)}+\alpha e_{12}^{(2)} \\
0 & \alpha
\end{array}\right]
$$

for some invertible element $a_{12}^{(1)} \in R$. By Proposition 2.2, we have that $e_{12}^{(1)}+e_{12}^{(2)}=0$ and

$$
\theta(A)=\left[\begin{array}{cc}
\alpha & b a_{12}^{(1)} \\
0 & \alpha
\end{array}\right]
$$

for some invertible element $a_{12}^{(1)} \in R$. Choosing $D=\left[\begin{array}{cc}1 & 0 \\ 0 & \left(a_{12}^{(1)}\right)^{-1}\end{array}\right] \in T_{2}(R)$, we can obtain that

$$
\theta(A)=D A D^{-1}
$$

which means that all the automorphisms of $S T_{2}(R)$ are inner. 
For the case $n=3$, we proceed with the steps similar to the case $n=2$ in order to determine the invertible matrix $B$.

Let $A=\left[\begin{array}{ccc}\alpha & b & c \\ 0 & \alpha & d \\ 0 & 0 & \alpha\end{array}\right] \in S T_{n}(R)$. By using matrix units, we get

$$
\begin{aligned}
& \theta\left(E_{11}\right)=\left(\begin{array}{ccc}
1 & e_{12}^{(1)} & e_{13}^{(1)} \\
0 & 0 & 0 \\
0 & 0 & 0
\end{array}\right) \\
& \theta\left(E_{22}\right)=\left(\begin{array}{ccc}
0 & e_{12}^{(2)} & e_{13}^{(2)} \\
0 & 1 & e_{23}^{(2)} \\
0 & 0 & 0
\end{array}\right) \\
& \theta\left(E_{33}\right)=\left(\begin{array}{ccc}
0 & 0 & e_{13}^{(3)} \\
0 & 0 & e_{23}^{(3)} \\
0 & 0 & 1
\end{array}\right) \\
& \theta\left(E_{12}\right)=\left(\begin{array}{ccc}
0 & a_{12}^{(1)} & a_{13}^{(1)} \\
0 & 0 & 0 \\
0 & 0 & 0
\end{array}\right) \\
& \theta\left(E_{23}\right)=\left(\begin{array}{ccc}
0 & 0 & a_{13}^{(2)} \\
0 & 0 & a_{23}^{(2)} \\
0 & 0 & 0
\end{array}\right)
\end{aligned}
$$

and we obtain

$$
\theta(A)=\left[\begin{array}{ccc}
\alpha & b a_{12}^{(1)} & c a_{12}^{(1)} a_{23}^{(1)}+b a_{13}^{(1)}+d a_{13}^{(2)}+\alpha\left(e_{13}^{(1)}+e_{13}^{(2)}+e_{13}^{(3)}\right) \\
0 & \alpha & d a_{23}^{(2)} \\
0 & 0 & \alpha
\end{array}\right] .
$$

Similarly, it can be deduced from Proposition 2.2,

$$
e_{13}^{(1)}+e_{13}^{(2)}+e_{13}^{(3)}=0 \Rightarrow \alpha\left(e_{13}^{(1)}+e_{13}^{(2)}+e_{13}^{(3)}\right)=0 .
$$

Hence,

$$
\theta(A)=\left[\begin{array}{ccc}
\alpha & b a_{12}^{(1)} & c a_{12}^{(1)} a_{23}^{(1)}+b a_{13}^{(1)}+d a_{13}^{(2)} \\
0 & \alpha & d a_{23}^{(2)} \\
0 & 0 & \alpha
\end{array}\right]
$$

It is now easy to define the invertible matrix $B \in T_{3}(R)$ as,

$$
B=\left(\begin{array}{ccc}
1 & a_{13}^{(1)}\left(a_{12}^{(1)} a_{23}^{(2)}\right)^{-1} & 0 \\
0 & \left(a_{12}^{(1)}\right)^{-1} & -a_{13}^{(1)}\left(a_{12}^{(1)}\right)^{2}\left(a_{23}^{(2)}\right)^{-1} \\
0 & 0 & \left(a_{12}^{(1)}\right)^{-1}\left(a_{23}^{(2)}\right)^{-1}
\end{array}\right)
$$

so that

$$
\theta(A)=\eta_{B}=B A B^{-1}
$$

Now, we can state the main theorem of this paper. 
Theorem 2.4. Let $\theta$ be an R-algebra automorphism of $S T_{n}(R)$ and $R$ be a commutative ring with identity. For $n \geq 4$,

$$
\theta=\eta_{D} \mu_{c} \lambda_{P}
$$

where $\eta_{D}, \lambda_{P}$ are inner automorphisms and $\mu_{c}$ is a central automorphism of $S T_{n}(R)$.

Proof. First, apply $\theta$ to each $E_{i i}$ for $i=1, \ldots,(n-1)$. The $S_{2}$ diagonal only contains the elements $e_{i, i+1}^{(p)}$. Thus we obtain

$$
\sum_{p=i}^{i+1} e_{i, i+1}^{(p)} E_{i, i+1} \text { for } p=1, \ldots, n-1 .
$$

By Proposition 2.2, we can get

$$
\sum_{p=i}^{i+1} e_{i, i+1}^{(p)}=0
$$

A consequence of above sum allows us just to see the image of $E_{i, i+1}$ under $\theta$. We examine

$$
\theta\left(E_{i, i+1}\right)=a_{i, i+1}^{(i)} E_{i, i+1} \text { for } i=1, \ldots, n-1,
$$

We want to show that $a_{p, p+1}^{(k)}=0$ if $k \neq p$. On the contrary, assume that $a_{p, p+1}^{(k)} \neq 0$ and take $k=1$ to get a contradiction. Apply $\theta$ to the equality $E_{p, p+1} E_{23}=0$, we obtain

$$
\begin{aligned}
\theta\left(E_{p, p+1} E_{23}\right) & =\theta\left(E_{p, p+1}\right) \theta\left(E_{23}\right) \\
{\left[a_{i j}^{(p)}\right]\left[a_{i j}^{(2)}\right] } & =0 .
\end{aligned}
$$

Consider the term $a_{12}^{(p)} a_{23}^{(2)} E_{13}$ on the left side of the above equality. Since $a_{12}^{(p)} a_{23}^{(2)}=0$ and $a_{23}^{(2)}$ is a unit by [3], then $a_{12}^{(p)}=0$. If $k>1$, apply $\theta$ to the equality $E_{k, k+1} E_{p+1, p+2}$ to get

$$
\left[a_{i j}^{(k)}\right]\left[a_{i j}^{(p+1)}\right]=0 .
$$

Consider the term $a_{p, p+1}^{(k)} a_{p+1, p+2}^{(p+1)} E_{p, p+2}=0$ on the left side of the above equality. Since $a_{p, p+1}^{(k)} a_{p+1, p+2}^{(p+1)}=0$ and $a_{p+1, p+2}^{(p+1)}$ is a unit then $a_{p, p+1}^{(k)}=0$ but, this is a contradiction. Now defining the diagonal matrix $D \in T_{n}(R)$ with diagonal entries from the set $\left\{1,\left(a_{12}^{(1)}\right)^{-1},\left(a_{12}^{(1)} \cdot a_{23}^{(2)}\right)^{-1}, \ldots,\left(a_{12}^{(1)} \cdot a_{23}^{(2)} \ldots a_{n-1, n}\right)^{-1}\right\}$ we have the following result on the diagonal $S_{2}$,

$$
\eta_{D}^{-1} \theta\left(E_{i, i+1}\right)=E_{i, i+1} \text { for } i=1, \ldots, n-1 .
$$

We are going to use induction on $t$ to prove that there exist inner automorphisms $\eta_{P_{t}}$ with $P_{t} \in T_{n}(R)$ and

$$
\eta_{P t}^{-1} \eta_{D}^{-1} \theta\left(E_{i, i+1}\right)=E_{i, i+1} \text { for } i=1, \ldots, n-1 \text { and } t=2, \ldots, n-1 .
$$

Assume that we have

$$
\eta_{P_{t}}^{-1} \eta_{D}^{-1} \theta\left(E_{i, i+1}\right)=E_{i, i+1}
$$

for $i=1, \ldots, n-1$, on the diagonals $S_{1}, \ldots, S_{t}$. What about on the diagonal $S_{t+1}$ ? Apply $\theta$ to $E_{i i}$, we can obtain the sum

$$
\sum_{p=i}^{t} e_{i, i+t}^{(p)} E_{i, i+t} \text { for } i=1, \ldots, n-t+1 .
$$

By Proposition 2.2, we have

$$
\sum_{p=i}^{t} e_{i, i+t}^{(p)} E_{i, i+t}=0
$$


Then we just consider the rest, that is:

$$
\eta_{P_{t}}^{-1} \eta_{D}^{-1} \theta\left(E_{i, i+1}\right)=E_{i, i+1}+\sum_{j=1}^{n-t} b_{j, i}^{(i)} E_{j, j+t} .
$$

We want to show $b_{j, i}^{(i)}=0$ if $j \neq i$ and $j \neq i-t+1$. Assume that $b_{p, q}^{(q)} \neq 0$ with $p \neq q$ with $p \neq q+1-t$. If $p<n-t$ then we apply $\eta_{P_{t}}^{-1} \eta_{D}^{-1} \theta$ to $E_{q, q+1} E_{p+t, p+t+1}$, we get $\eta_{P_{t}}^{-1} \eta_{D}^{-1} \theta\left(E_{q,, q+1} E_{p+t, p+t+1}\right)=0$. Thus, we obtain the following results:

$$
\left[E_{q, q+1}+\sum_{j=1}^{n-t} b_{j, q}^{(q)} E_{j, j+t}\right]\left[E_{p+t, p+t+1}+\sum_{j=1}^{n-t} b_{j, p+t}^{(p+t)} E_{j, j+t}\right]=0
$$

and

$$
b_{q+1, p+t}^{(p+t)} E_{q, p+t+1}+b_{p, q}^{(q)} E_{p, p+t+1}=0 .
$$

Hence $b_{p q}^{(q)}=0$, we get a contradiction. If $p=n-t$ then apply $\eta_{P_{t}}^{-1} \eta_{D}^{-1} \theta$ to $E_{p-1, p} E_{q, q+1}$ we get

and

$$
\eta_{X}^{-1} \eta_{D}^{-1} \theta\left(E_{p-1, p} E_{q, q+1}\right)=0
$$

$$
b_{p q}^{(q)} E_{p-1, p+t}+b_{q-t, p-1}^{(p-1)} E_{q-t, q+1}=0 .
$$

Since $b_{p q}^{(q)}=0$, we get a contradiction again. Hence, on the $S_{t+1}$ diagonal we have

$$
\eta_{P_{t}}^{-1} \eta_{D}^{-1} \theta\left(E_{i, i+1}\right)=E_{i, i+1}+b_{i-t+1, i}^{(i)} E_{i-t+1, i+1}+b_{i, i}^{(i)} E_{i, i+t} .
$$

However, we want to get $\eta_{P_{t}}^{-1} \eta_{D}^{-1} \theta\left(E_{i, i+1}\right)=E_{i, i+1}$.

To prove this, we use induction again. Assume that there exists an inner automorphism $\lambda_{G_{k-1}}$ such that on the $S_{t+1}$ diagonal:

$$
\lambda_{G_{k-1}}^{-1} \eta_{P_{t}}^{-1} \eta_{D}^{-1} \theta\left(E_{i, i+1}\right)=E_{i, i+1} \text { for } i=1, \ldots, k-1,
$$

and

$$
\lambda_{G_{k-1}}^{-1} \eta_{P_{t}}^{-1} \eta_{D}^{-1} \theta\left(E_{i, i+1}\right)=E_{i, i+1}+d_{i-t+1, i}^{(i)} E_{i-t+1, i+1}+d_{i, i}^{(i)} E_{i, i+t} \text { for } i=k, \ldots, n-1 .
$$

Setting $Z=I+d_{k-t+1, k}^{(k)} E_{k-t+1, k}-d_{k, k}^{(k)} E_{k, k+t}$ and $G_{k}=G_{k-1} Z$ then for $1 \leq i<k$ we have

$$
\lambda_{G_{k}}^{-1} \eta_{P_{t}}^{-1} \eta_{D}^{-1} \theta\left(E_{i, i+1}\right)=E_{i, i+1}+\delta_{i, k-t} d_{k+1-t, k}^{(k)} E_{i, k}
$$

on the $S_{t+1}$ diagonal. For $i=k-t$, applying $\lambda_{G_{k-1}}^{-1} \eta_{P_{t}}^{-1} \eta_{D}^{-1} \theta$ to the equality $E_{k-t, k-t+1} E_{k, k+1}=$ 0 , we obtain

on the diagonal $S_{t+1}$. Hence

$$
d_{k+1-t, k}^{(k)} E_{k-t, k+1}=0
$$

We get

$$
d_{k+1-t, k}^{(k)}=0 .
$$

$$
\lambda_{G_{k}}^{-1} \eta_{P_{t}}^{-1} \eta_{D}^{-1} \theta\left(E_{k, k+1}\right)=E_{k, k+1}
$$

for $i=1, \cdots, k$. For $i>k$, as stated on [1], we have

$$
\lambda_{G_{k}}^{-1} \eta_{P_{t}}^{-1} \eta_{D}^{-1} \theta\left(E_{i, i+1}\right)=E_{k, k+1}+\left(d_{i-t+1, i+1}^{(i)}+\delta_{k+t, i} d_{k k}^{(k)}\right) E_{i+1-t, i+1}+d_{i i}^{(i)} E_{i, i+t} .
$$

Thus, there exists an inner automorphism $\lambda_{P_{n-1}}$ of $S T_{n}(R)$ such that

$$
\lambda_{P_{n-1}}^{-1} \eta_{D}^{-1} \theta\left(E_{i, i+1}\right)=E_{i, i+1}
$$

on the $S_{n-1}$ diagonal. We want to know what happens on the $S_{n}$ diagonal. Assume that, we have

$$
\lambda_{P_{n-1}}^{-1} \eta_{D}^{-1} \theta\left(E_{i, i+1}\right)=E_{i, i+1}+c_{i} E_{1 n} \text { for } i=1, \ldots, n-1
$$


on the $S_{n}$ diagonal. If we apply $\lambda_{P_{n-1}}^{-1} \eta_{D}^{-1} \theta$ to $E_{i i}$, we get

$$
\left(e_{1 n}^{(1)}+e_{1 n}^{(2)}+\ldots+e_{1 n}^{(n-1)}\right) E_{1 n} .
$$

By Proposition 2.2, $\left(e_{1 n}^{(1)}+e_{1 n}^{(2)}+\ldots+e_{1 n}^{(n-1)}\right)=0$ and if we take

$$
Z=I+c_{n-1} E_{1, n-1}-c_{1} E_{2 n}
$$

as in [1], we get

$$
\lambda_{Z}^{-1} \lambda_{p_{n-1}}^{-1} \eta_{D}^{-1} \theta\left(E_{i, i+1}\right)=E_{i, i+1} \text { for } i=1, n-1
$$

and

$$
\lambda_{Z}^{-1} \lambda_{P_{n-1}}^{-1} \eta_{D}^{-1} \theta\left(E_{i, i+1}\right)=E_{i, i+1}+c_{i} E_{1 n} \text { for } i=2, \ldots, n-2 .
$$

Setting $c=\left(c_{2}, \ldots, c_{n-2}\right)$, we get

$$
\mu_{c}^{-1} \lambda_{Z}^{-1} \lambda_{p_{n-1}}^{-1} \eta_{D}^{-1} \theta\left(E_{i, i+1}\right)=E_{i, i+1} \text { for } i=1, \ldots, n \text {. }
$$

So we have

$$
\mu_{c}^{-1} \lambda_{Z}^{-1} \lambda_{p_{n-1}}^{-1} \eta_{D}^{-1} \theta=1
$$

Thus

$$
\theta=\eta_{D} \mu_{c} \lambda_{P}
$$

for some $P \in T_{n}(R)$.

Remark 2.5. Let $N_{n}(R)$ be the strictly upper triangular matrix algebra over $R$, then

$$
\operatorname{Aut}\left(N_{n}(R)\right) \simeq \operatorname{Aut}\left(S T_{n}(R)\right) \text {. }
$$

Example 2.6. Let $A \in S T_{4}(R)$ be defined as $\left[\begin{array}{cccc}a & b & c & d \\ 0 & a & e & f \\ 0 & 0 & a & r \\ 0 & 0 & 0 & a\end{array}\right]$.

We can check that $\theta: S T_{4}(R) \rightarrow S T_{4}(R)$ defines an automorphism via

$$
\theta\left(\left[\begin{array}{cccc}
a & b & c & d \\
0 & a & e & f \\
0 & 0 & a & r \\
0 & 0 & 0 & a
\end{array}\right]\right)=\left[\begin{array}{cccc}
a & b & c & d-3 e \\
0 & a & e & f \\
0 & 0 & a & r \\
0 & 0 & 0 & a
\end{array}\right]
$$

But $\theta$ is not an inner automorphism. Notice that $\operatorname{Aut}\left(S T_{n}(R)\right) \nsubseteq A u t\left(T_{n}(R)\right)$. Then all the automorphisms of $S T_{n}(R)$ cannot be extended to the automorphisms of $T_{n}(R)$.

\section{References}

[1] Y. Cao and J. Wang, A note on algebra automorphisms of strictly upper triangular matrices over commutative rings, Linear Algebra Appl. 311, 187-193, 2000.

[2] S.P. Coelho, Automorphism group of certain algebras of triangular matrices, Arch. Math. 61, 119-123, 1993.

[3] T.P. Kezlan, A note on algebra automorphisms of triangular matrices over commutative rings, Linear Algebra Appl. 135, 181-184, 1990. 